\title{
Pyonephrosis drained by double-J catheter
}

\author{
Che-Wei Chang ${ }^{1}$ and Chun-Nung Huang ${ }^{2}$ \\ ${ }^{1}$ Kaohsiung Medical University \\ ${ }^{2}$ Kaohsiung Municipal Ta-Tung Hospital
}

May 14, 2020

\begin{abstract}
A 74-year-old female with cervical cancer post radical hysterectomy developed bilateral ureter stricture with pyonephrosis. Retrograde ureteric stent was performed and it was considered an effective procedure other than percutaneous nehrostomy and nephrectomy.
\end{abstract}

Pyonephrosis drained by double-J catheter

Che-Wei Chang ${ }^{1,2,3}$, Chun-Nung Huang ${ }^{1,4}$

1. Department of Urology, School of Medicine, College of Medicine, Kaohsiung Medical University, Kaohsiung, Taiwan

2. Department of Urology, Kaohsiung Medical University Hospital, Kaohsiung, Taiwan

3. Department of Urology, Kaohsiung Municipal Siaogang Hospital, Kaohsiung, Taiwan

4. Department of Urology, Kaohsiung Municipal Ta-Tung Hospital, Kaohsiung, Taiwan

Correspondence to Chun-Nung Huang, M.D. Ph.D.

Department of Urology, School of Medicine, College of Medicine, Kaohsiung Medical University

Department of Urology, Kaohsiung Municipal Ta-Tung Hospital, Kaohsiung, Taiwan

E-mail: cnhuang.uro@gmail.com

\section{Keywords}

Pyonephrosis, hydronephrosis, double-J catheter, ureteric stent

\section{Key Clinical Message}

Pyonephrosis is an infectious disease with obstructive hydronephrosis, causing suppurative destruction of the renal parenchyma. Retrograde ureteric stent is a good choice for drainage of pyonephrosis other than percutaneous nehrostomy and nephrectomy

\section{Case summary}

- A 74-year-old female had cervical cancer post radical hysterectomy, lymphadenectomy and salpingooophorectomy. Bilateral ureter stricture with regular double-J catheter revision was impressed. The latest bilateral double-J catheter revision was performed three months ago. Abdomen computational tomography revealed persistent bilateral hydronephrosis (Fig 1). Thus, bilateral double-J revision was performed. Cystoscopy shown turbid urine with right double-J catheter dislodged. After replacement of 
the right double-J catheter, pus like material effluxed from side hole of double-J stent which confirmed the diagnosis of pyonephrosis (Fig 2, Video).

- Pyonephrosis is an infectious disease usually accompanied with obstructive hydronephrosis, causing suppurative destruction of the renal parenchyma. Retrograde ureteric stent is a good choice for drainage of pyonephrosis other than percutaneous nehrostomy and nephrectomy [1].

\section{Ethics approval and consent to participate}

Approval for the study was obtained from the institutional review board of Kaohsiung Municipal Ta-Tung Hospital

\section{Consent for publication}

Informed consent was obtained from the patient for the publication of this case report.

\section{Availability of data and materials}

The authors do not wish to share the patient's data. The privacy of this participant should be protected.

\section{Competing interests}

The authors declare no conflicts of interest.

\section{Funding}

No funding sources contributed to this case report.

\section{Authors' contributions}

Chun-Nung Huang performed the surgery. Che-Wei Chang reviewed this case, and was a major contributor in writing the manuscript. All authors read and approved the final manuscript.

\section{Reference}

1. Flukes, S., et al., Retrograde ureteric stent insertion in the management of infected obstructed kidneys. BJU Int, 2015. 115 Suppl 5 : p. 31-4.

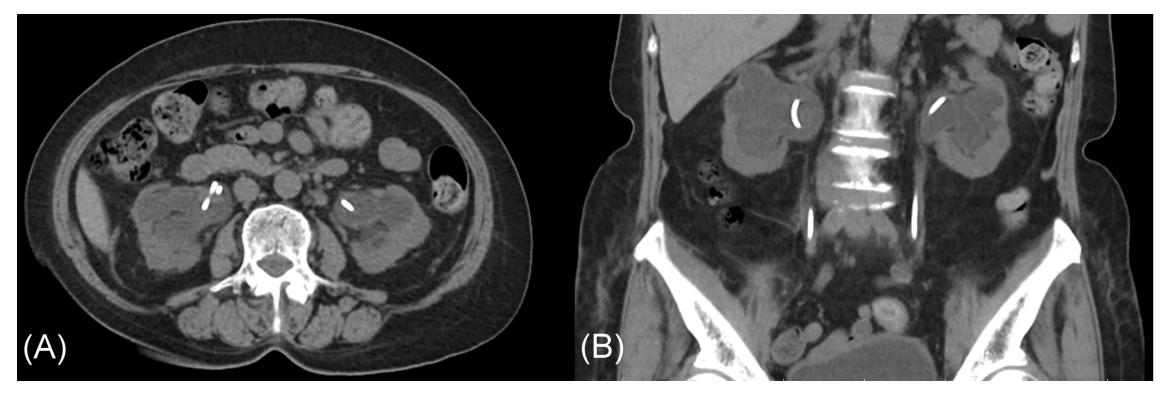




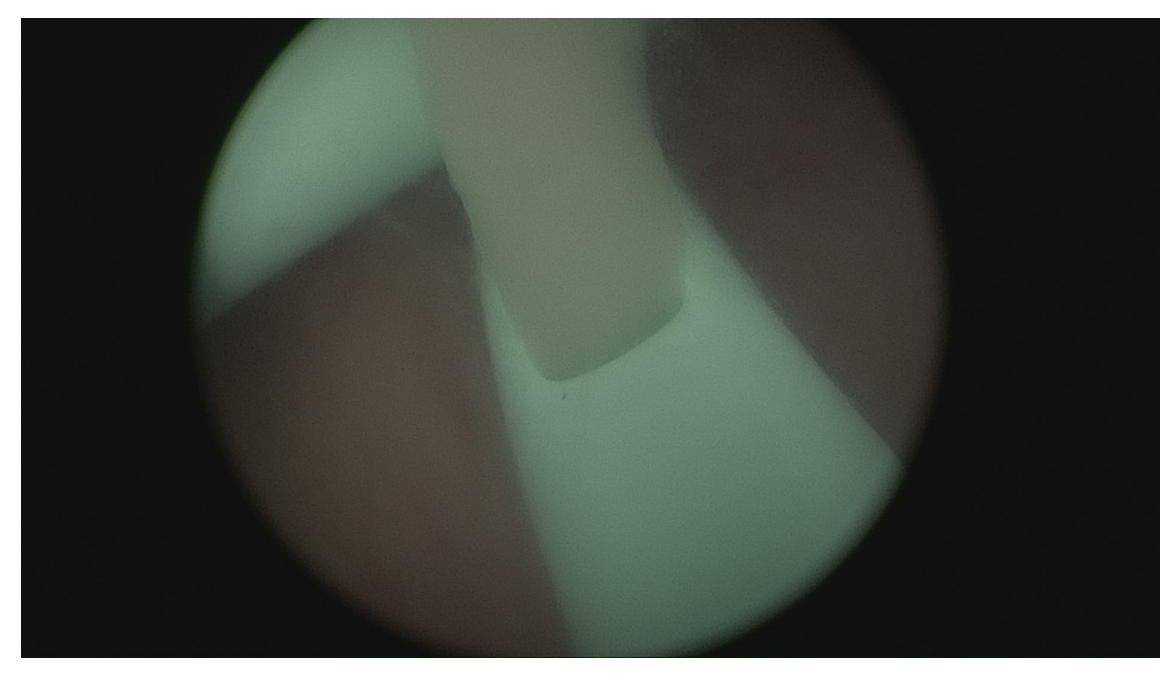

